\title{
Pratiques littératiées et construction des inégalités : une étude de cas dans l'enseignement professionnel agricole
}

\author{
LAURE MINASSIAN \\ CIRCEFT-Escol, Université Paris 8 et Université de Caen
}

Résumé

De nombreuses études analysent le rôle des supports de travail proposés aux élèves dans la construction des inégalités. Mais très peu d'entre elles s'intéressent à l'enseignement professionnel, moins encore agricole. Cette étude inédite cherche à documenter ces enseignements et s'inspire des New Literaties Studies pour mettre en évidence quelques relations entre littératies et inégalités. Le courant des new litteraties studies invite à la mobilisation d'objets disciplinaires différents pour étudier un même phénomène. Dans le cas précis, les notions de « littératie du travail » et « littératie scolaire » sont mobilisées pour comprendre comment, en bac pro, ces univers sont reconfigurés. L'étude compare deux classes de même niveau, situées dans des établissements contrastés. L’analyse des productions des élèves permet d'interroger l'impact de deux orientations pédagogiques selon la socialisation des élèves.

\section{Mots-clés}

littératie, inégalités sociales et scolaires, enseignement professionnel

\section{Introduction}

La notion de littératie invite à la mise en relation entre écrits et inégalités quand on sait l'importance de l'écrit dans les formes de transmission où qu'elles se trouvent, que ce soit en contexte scolaire (Delarue-Breton \& Bautier, 2015) dans le domaine de la santé (Balcou-Debussche, 2016), du travail (Boutet, 2001), ou encore domestique (Lahire, 1995). La multiplication et la diversification des lieux où s'exerce la littératie constituent un phénomène qui intéresse les sciences sociales depuis une période récente.

On doit en grande partie à l'ethnographie et l'anthropologie la prise en compte d'éléments hétérogènes pour décrire les pratiques sociales dans lesquelles s'actualisent les littératies. L'étude simultanée des littératies urbaines et rurales a permis de décrire l'émergence de l'écriture au Mexique (Rockwell, 2010), la mise en relation des compétences culturelles et sociales s'est avérée fructueuse pour penser les rapports à l'écrit en Namibie (Papen, 2010). L’étude présente, centrée sur l'enseignement agricole, s’inspire de ces travaux. Ni académique ni sphère du travail productif, cet enseignement se situe dans un entre-deux, un espace de recompositions et d'hybridations de deux sphères habituellement séparées.

Il faut ajouter que l'étude des littératies dans l'enseignement professionnel et agricole est peu fréquente. Alors que de nombreux travaux apportent un éclairage indispensable pour la compréhension des phénomènes dans l'écrit qui participent aux inégalités d'apprentissage, notamment au travers de la mise en évidence d’implicites (Bautier \& Rochex, 1998 ; Viriot-Goeldel \& Delarue-Breton, 2014), ils restent néanmoins 
cantonnés à l'enseignement général. De ce fait, on ne dispose pas d'une représentation qui permette de mesurer l'intensité des mêmes phénomènes et leurs nuances dans l'enseignement professionnel. On peut faire l'hypothèse que cette absence n'est pas sans lien avec une image fréquente selon laquelle l'écrit dans ces segments d'enseignements serait moins répandu et moins exigeant. Au contraire, notre étude montre qu'il y est très répandu et d'une autre exigence.

Notre propos s'appuie sur deux études de cas et vise à objectiver, dans deux classes de même niveau, mais dont les pratiques pédagogiques et les publics diffèrent, ce qui se joue dans la manière de présenter des contenus. Le propos s'inscrit tout d'abord dans une problématique d'ordre sociologique autour de la question des inégalités d'apprentissage. Ensuite, le détour notionnel permettra de montrer la fécondité d'un cadre conceptuel construit autour de la notion de littératie. Ces éléments au soubassement de la méthodologie adoptée autorisent l'analyse des supports pédagogiques où s'articulent différentes sphères : professionnelle, scolaire et scientifique. Enfin, les effets de ces supports seront analysés à partir des productions écrites des élèves.

\section{Problématique}

Notre propos s'inscrit dans les recherches de l'équipe EScol (Education Scolarisation), dont les objets d'étude relèvent des inégalités sociales, de l'appropriation des savoirs scolaires et de ce qui se joue dans la confrontation des pratiques des enseignants et des interprétations des élèves. Il s'agit alors de mettre au jour un ensemble de phénomènes qui participent de mésinterprétations, de malentendus. Notre propos, s’inscrit précisément dans ces thématiques qui relèvent tout à la fois des inégalités - qui d'ailleurs peuvent contribuer de différenciations pédagogiques - et de la difficulté. Les résultats de l'équipe montrent que les inégalités d'apprentissage résultent pour une part de la confrontation entres des socialisations d'élèves et le caractère implicite, parfois opaque des attentes des enseignants. Cette hypothèse relationnelle nous a conduit ainsi à mettre l'accent sur certains modes de faire de la part des enseignants, qui d'ailleurs peuvent être en relation avec des difficultés perçues, qui peuvent avoir pour effet d'accroître ces difficultés à l'inverse même de l'intention de départ. Les filières du baccalauréat professionnel ou agricole ne font pas figures d'exception.

En portant l'attention sur l'ordinaire de la classe, les modalités concrètes des pratiques d'enseignement et d'apprentissage, s'élabore une problématique d'ordre sociologique attentive à l'analyse d'univers et de savoirs présentés dans des supports pédagogiques destinés aux élèves et ceux que ces derniers mobilisent effectivement dans leurs copies. Les paragraphes qui suivent présentent le cadre théorique et conceptuel à partir duquel sont analysés des processus à l'œuvre dans la production des inégalités d’apprentissage des élèves au sein de l'enseignement agricole.

\section{Cadre théorique La littératie, contextes et dispositions des sujets}

L'enseignement agricole constitue un objet intermédiaire entre l'école et le travail productif et scolarise des élèves aux parcours socio-scolaires hétérogènes (du fils d'agriculteur assuré d'une reprise de l'exploitation parental, au fils d'ouvrier évoluant dans un contexte urbain sans lien avec le monde rural). Le cadre théorique retenu rend compte de ces spécificités à double titre. Tout d'abord, l'inscription de l'objet dans le champ de recherche des new litteraties studies, donne à voir cette formation comme espace de 
transaction à la croisée de mondes pluriels. Ensuite, l'étude centrée d'une part sur les supports de cours rédigés par les enseignants et d'autre part sur ce que manifestent les pratiques scripturales des élèves, relève d'un cadre général où l'écrit est considéré comme un outil à partir duquel des pratiques de sens sont élaborées. Ces dernières peuvent être éloignées de celles initialement visées du fait entre autres qu'elles se construisent dans l'interaction avec des pairs, une expérience antérieure, des pratiques de stages, etc. Cette perspective double permet alors de tenir ensemble des présentations de savoirs sociotechniques complexes et des interprétations socialement situées d’élèves.

\section{La littératie dans l'enseignement agricole : à la croisée de mondes pluriels}

L'un des intérêts pour notre enquête de recourir au courant anglo-saxon des new litteraties studies est qu'il met l'accent 1/sur les usages des documents graphiques en contexte et 2/sur les significations multiples que peuvent prendre ces derniers selon les individus qui y sont confrontés :

«[les littératies] se fusionnent aux contextes sociopolitiques, culturels, économiques, genrés et racialisés qui les encodent. Ces contextes ne sont pas statiques. Ils sont fluides et transforment les littératies qui produisent des locuteurs, des scripteurs, des artistes, des communautés. Ces actes de construction de sens qu’on qualifie de littératies sont intégrés à la culture et aux dimensions sociopolitiques et socio-historiques d'une société et de ses institutions. Le sens de littératies s'opérationnalise ou s'actualise à partir d'un contexte particulier dans le temps et dans l'espace où il se trouve et opère » (Masny \& Dufresne, 2007, p. 215).

Cette dimension incite au dépassement d'une analyse par discipline et sous-discipline de recherche comme l'indiquent Fraenkel et Mbodj (2010) au profit de la prise en compte d'une pluralité d'éléments indissociables, mais disciplinairement hétérogènes, en vue de comprendre une même réalité (Barton \& Hamilton, 2010). Ces réflexions trouvent un point d'achoppement avec les segments de l'enseignement professionnel et agricole dont l'une des spécificités relève de la co-présence de deux sphères sociales : l'école et le stage. Dans chacune de ces sphères, l'écrit ne vise pas les mêmes finalités. Alors que dans la sphère productive, ils peut être en lien avec la recherche de l'efficience au regard des ressources investies, la réactivité à l'égard de la demande ou l'innovation dans la transformation des objets par exemple (Boutet, Gardin \& Lacoste, 1995) ; à l'école, en particulier dans le second degré, l'écrit est très fréquemment un écrit long d'élaboration, il répond à des consignes et vise à produire des textes descriptifs, argumentatifs, analytiques, dans l'objectif d'une évaluation scolaire.

Ni strictement conformes au modèle traditionnel scolaire (academic litteracy), ni à celui du travail (workplace litteracy), les pratiques écrites dans l'enseignement agricole, et plus généralement professionnel, n’ont pas vocation à agir directement sur le travail productif, il s'agit plus modestement de construire des modes de raisonnement de surplomb, en vue de préparer les élèves à l'exercice du métier. Cette attente est d'ailleurs 
soulignée à de multiples reprises dans le référentiel de formation ${ }^{1}$ comme le montrent les quelques extraits ci-dessous :

- «but : l'acquisition par les apprenants de méthodes permettant l'analyse dans une approche systémique du fonctionnement d'une entreprise agricole »

- «Repérer les attentes sociétales vis-à-vis de l'agriculture. Cet objectif constitue l'occasion d'aborder : la place de l'agriculture dans la société (1), les débats sur l'agriculture (2)»

- «Dans cet objectif, les « ressources » doivent être prises dans une acception large. Elles concernent aussi bien les ressources «naturelles » et les processus écologiques que les leviers techniques, les savoirs scientifiques (académiques ou locaux) ou les ressources « humaines » que l'on peut mobiliser dans le fonctionnement des agroécosystèmes »

(Référentiel de formation bac pro CGEA, 2017, p. 36 ; 57 ; 61).

En ce sens, les supports sur lesquels se base la présente étude sont des objets hybrides, construits à partir de deux mondes sociaux distincts et parfois distants (Latour \& Woolgar, 1988).

La littératie comme activité de sens non prévisible : la question de l'interprétation

Ces spécificités socio-techniques d'une part et scolaires de l'autre, invitent à s'intéresser au sens qu'en construisent les élèves. Si la présentation des savoirs constitue une condition nécessaire pour cette élaboration, elle est toutefois non suffisante. Les travaux de Scribner et Cole (1973) montrent que la maitrise formelle des outils de la littératie (la cartographie, la numératie, ou encore l'iconographie, la partition de musique) ne donne pas nécessairement lieu à l'entrée dans l'univers de la littératie. Cette perspective est également défendue par Heath et Street (2008) qui considèrent l'appropriation du sens, toujours hétérogène, partielle, comme la résultante de processus d'interactions entre un contexte, des ressources symboliques et les modes de pensée auxquels sont familiarisés les individus. Ces développements sur la notion rejoignent également les travaux de Hennion sur la question de l’interprétation (2007 [1993]) :

« la médiation, comprise exactement ainsi, (...) n’importe quel musicien qui se met au clavier sait qu'il a ses gammes, sa partition, son toucher, les tours de main qu'il a acquis, qu'il ne fait rien sans eux, mais que pourtant, partant de ces médiations, rien n’est fait, il va falloir faire surgir la musique et il n’y a rien là qui soit automatique ou garanti » (Hennion, 2013).

${ }^{1}$ D’un point de vue politique, le baccalauréat professionnel a été créé en 1985 au moment de ce que l'on a appelé la seconde vague de massification scolaire (Beaud, 2003). Sa mise en place coïncide avec l'objectif énoncé par Chevènement, alors ministre de l'Education Nationale, de permettre à $80 \%$ d'une génération d'accéder au baccalauréat. Sur le plan de l'organisation, le curricculum du baccalauréat, quel que soit sa spécialité, relève de deux référentiels : le référentiel professionnel, dont l’objectif vise à identifier les compétences nécessaires pour exercer une profession spécifique, et le référentiel de formation, lequel a pour objet la scolarisation des compétences de métier, afin de permettre leur acquisition au travers de la maitrise de méthode de raisonnement et d'objets de savoirs. 
Ces détours théoriques sont d'importance pour l'analyse des supports graphiques écrits qui nous intéressent. Ces derniers participent à produire quelque chose qui comporte des effets imprévisibles, "non déductibles d'une addition de facteurs causaux » (Hennion, 2013, §12). C'est la raison pour laquelle l'intérêt est porté sur des pratiques de sens plus ou moins en conformité avec les attentes du référentiel de formation et du métier au regard de ces supports. Ces attentes de l'école ont partie liée avec la mise en œuvre de la part des élèves d'une posture distanciée, essentielle étant donné la distance qui sépare l'expérience individuelle et les ambitions professionnelles actuelles.

\section{Les objectifs de la recherche}

Un des objectifs de ce travail vise à identifier ce que les élèves interprètent des objets de savoirs auxquels ils sont confrontés : il s'agit d'apprendre à l'école des modes de raisonnement qui n'en relèvent pas complètement et qui ne sont pas non plus tout à fait ceux du travail. Comment alors les enseignants enseignent-ils l'agriculture, comment présentent-ils des contenus sur le travail ? Quels éléments valorisent les élèves ?

On le comprend aisément, les présentations de savoirs dans ce segment de l'enseignement peuvent générer de nombreux malentendus en particulier lorsque le hiatus entre ce que l'enseignant présuppose des acquisitions cognitives des élèves, et celles que réalisent effectivement ces mêmes élèves est grand.

Des leviers ont déjà été identifiés par les nombreuses recherches qui portent sur littératies et inégalités dans l'école (Bonnéry \& Joigneaux, 2015 ; Laparra \& Margolinas, 2012). Elles ont montré les nombreux implicites face à un texte, un document, une image que peinent à identifier les élèves en particulier les plus éloignés des réquisits scolaires. Cette préoccupation ici transposée à l'enseignement professionnel et agricole, porte une acuité nouvelle du fait de la spécificité même de l’objet : un objet ni-ni, c'est-à-dire ni scolaire, ni du travail.

En centrant l'analyse sur les documents supports de travail des élèves, des recompositions auxquelles ces documents donnent lieu, quelques logiques pédagogiques à l'œuvre et ce qu'elles produisent chez les élèves en termes de malentendus peuvent être identifiées.

\section{Contexte et Méthodologie}

\section{Contexte de l'enquête}

Les observations de classe ont eu lieu dans deux établissements de l'Ouest de la France, dans deux classes de même niveau conduisant au même diplôme : le bac pro agricole (Conduite et Gestion d'une Exploitation Agricole). Au moment de l'enquête, les élèves ont majoritairement entre 17 et 18 ans et constituent des classes d'environ 17 élèves.

Dans le contexte français, l'enseignement agricole relève d'une formation en alternance, laquelle confronte les élèves à au moins deux lieux différents de socialisation. Cette confrontation favorise potentiellement un décloisonnement entre ce qui est de l'ordre scolaire et non-scolaire de la part des élèves comme des enseignants.

Le cours observé se situe justement à la jonction du travail et de l'école. Plus précisément, il s'agit de faire du stage un lieu de reprise de l'expérience propre pour en dégager des savoirs transférables, adaptables, généralisables. Les deux établissements (nommés dans la suite du texte école 1, école 2) se différencient par leur histoire. À 
l'origine, l'école 1 était plus fréquemment destinée à la petite paysannerie ; par contraste, l'école 2 était plutôt destinée à la grande paysannerie. Aujourd'hui l'enseignement agricole est moins fréquenté par les fils d'agriculteurs qu'autrefois: en 20 ans, la moitié des exploitations agricoles ont disparu (Butault \& Delame, 2003), conduisant à la raréfaction du public traditionnel. De ce fait, de nouveaux élèves aux parcours socio-scolaires plus hétérogènes ont intégré cette formation et ces mêmes élèves sont moins familiarisés aux raisonnements attendus dans le travail que par le passé, puisque leurs parents n’appartiennent pas toujours à ce secteur professionnel.

Cette tendance n'est pas homogène pourtant. Alors qu'ils ont connu des parcours scolaires plutôt ordinaires dans l'école 2 où ils sont plutôt fils d'agriculteurs; dans l'école 1, les élèves socialement hétérogènes ont davantage été marqués par l'échec scolaire, le redoublement, les orientations précoces, pour certains le décrochage, et sont majoritairement fils d'ouvriers non agricole.

Méthodologie. Le cas précis articule deux études de cas, dans la lignée des travaux de Glaser et Strauss (1967). L’observation intensive a autorisé à explorer des phénomènes complexes et peu connus sur un empan temporel long, 6 mois par classe. La prise en compte des écrits a permis d'identifier des récurrences qui font obstacles aux apprentissages des élèves. L'analyse est centrée sur l'objectivation des types d'hybridation des supports scolaires avec des univers de savoirs. Car une des tâches majeures des enseignants consiste à présenter graphiquement des savoirs en concevant, sélectionnant, recomposant, reprenant des ressources pédagogiques à destination des élèves et sur lesquels ils s'appuient pour dérouler la séance.

Une analyse détaillée des supports portant sur l'alimentation des vaches laitières au regard de leur production, c'est-à-dire la partie du référentiel la plus traditionnelle de cet enseignement (Denis \& Théret, 1994) permet de dégager des caractéristiques. L’analyse porte sur les contenus tout autant que sur un ensemble de procédés sémiotiques qui permettent de rendre compte les logiques pédagogiques contrastées, notamment au travers de la valorisation de certains éléments au détriment d'autres, de listes par puces, mises en retrait, paragraphes continus, tableaux, encadrés, signes graphiques, titres, soulignements, types de caractères, etc. La mise au premier plan des éléments présentés comme les plus pertinents, les formes linguistiques que prennent les consignes, en particulier les formes verbales et averbales, et les types d'écrit attendus qu'elles supposent (un texte argumentatif, descriptif, liste d'indicateurs par exemple), sont autant d'indices pris en compte.

Ensuite du point de vue des écrits des élèves, il s’agit de cumuler des éléments sur ce qui semble faire sens pour eux. On utilisera la notion de "pratiques langagières " développées par Boutet (1980), Benoît, Ebel et Fiala (1984), et Bautier (1995). Plus précisément, il s’agit d’analyser les énoncés langagiers comme pratiques sociales : « [les pratiques langagières] interviennent en tant que telles dans les domaines de la vie sociale et avec des effets matériels qui sont liés directement à leur exercice. [...] [elles] ont, dans leur spécificité, des effets sociaux immédiats liés à leurs formes mêmes et aux conditions dans lesquelles elles s'exercent. » (Benoît et al., 1983).

Les extraits des écrits d'élèves présentés dans le cadre de ce propos sont illustratifs de récurrences. Ils portent sur deux sujets conjoints : la production de lait et le contrôle des cycles de reproduction et font apparaître des différences qualitatives significatives sur les 
plans de l'organisation formelle des énoncés et de références aux savoirs d'expérience du métier, quant au contenu.

\section{Résultats}

L'organisation des supports pédagogiques : des littératies du travail agricole à l'école d'agriculture

Une des différences notables entre les deux classes relève de logiques pédagogiques contrastées. Alors que la pédagogie dans la classe de l'école 1 est davantage centrée sur l'activité productive pour construire presque directement des modes de pensée propres à un exploitant agricole, celle présente dans la classe de l'école 2 relève davantage d'une scolarisation du travail avec un découpage des savoirs selon des degrés de complexité.

Ecole 1. Une littératie du travail ordonnée selon l'organisation visuelle et temporelle des exploitations. Dans l'école 1, chaque support pédagogique destiné aux élèves est l'occasion de visiter un ensemble d'entités qui composent une exploitation agricole. Le support correspondant au chapitre de l'alimentation des vaches laitières participe de cette tendance, et comprend un ensemble de domaines à explorer, faisant du thème principal un objet présent de façon exclusive sous des formes implicites (cf. extrait 1). 


\section{Extrait 1.}

\section{Support école 1 : l'organisation de l'exploitation}

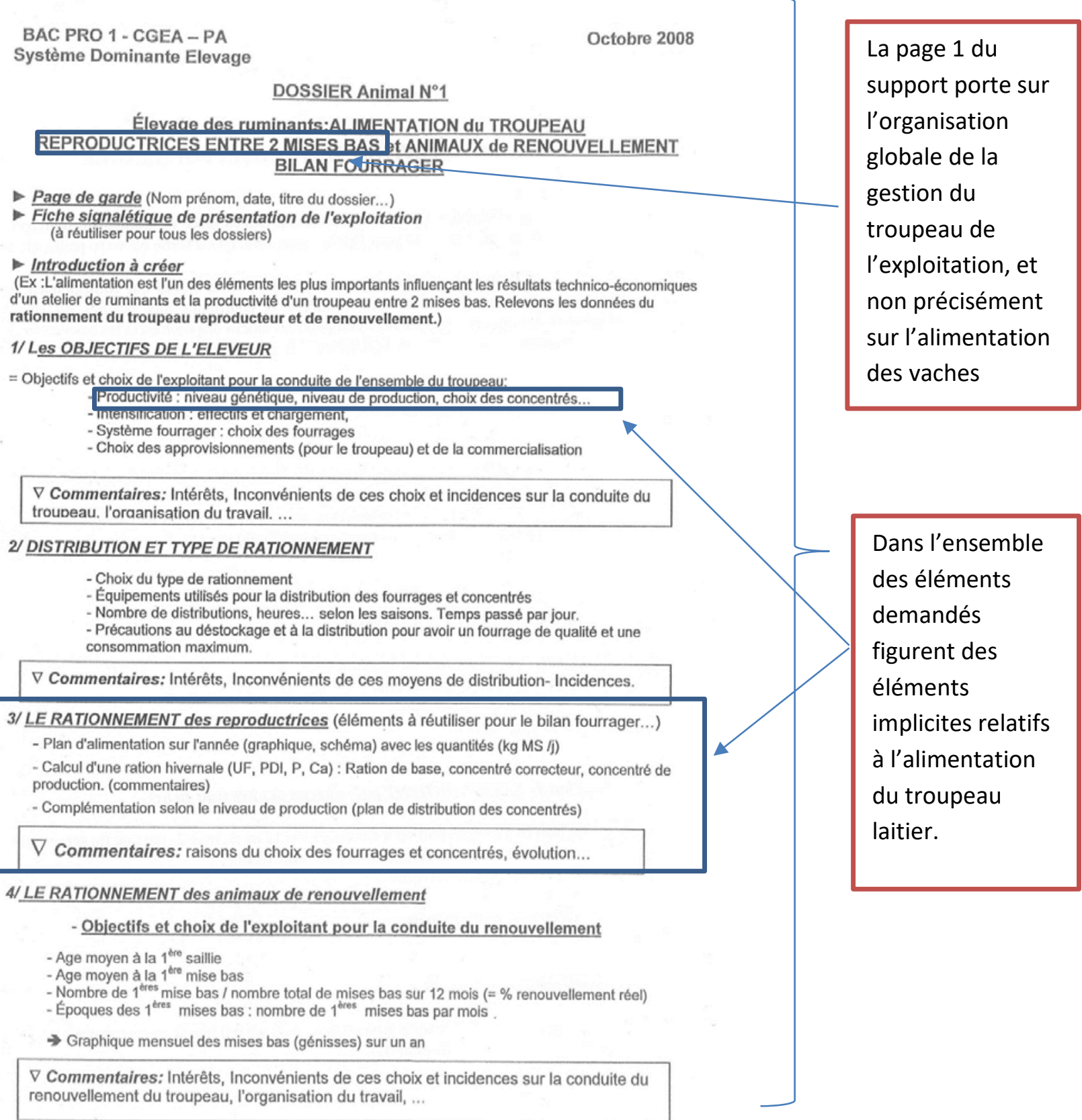

Ainsi, dans l'extrait ci-dessus, il s'agit de prendre en compte l'exploitation dans son ensemble, et non le troupeau laitier seulement. Sur l'extrait ci-après figure un exercice de calcul des stocks de fourrages disponibles (par cubage des silos, par pesée géométrique, cf. extrait 2 et annexes) sur l'ensemble de l'exploitation. La procédure de calcul du fourrage 
est donnée en vrai au sens où il semble que les élèves soient attendus à mesurer en unité cubique le stock existant, ce qui est particulièrement complexe, tout comme à estimer la quantité de fourrage à venir par une estimation de l'herbe sur pied (voir annexes). Autrement dit, c'est moins un exercice scolaire, au sens du référentiel, "acquérir une méthode d'analyse systémique », que d'acquérir des formes de raisonnements localement situées. Ce type de pédagogie, complexe puisqu'il s’agit de partir de phénomènes concrets, d'observations pour remonter en généralité, est une revendication de l'enseignant et une culture d'établissement. On peut d'ailleurs louer une logique pédagogique originale et pertinente. Ce qui nous intéresse cependant c'est ce qu'elle produit pour les élèves, nous y reviendrons.

\section{Extrait 2.}

Support école 1 : calcul des stocks de fourrages

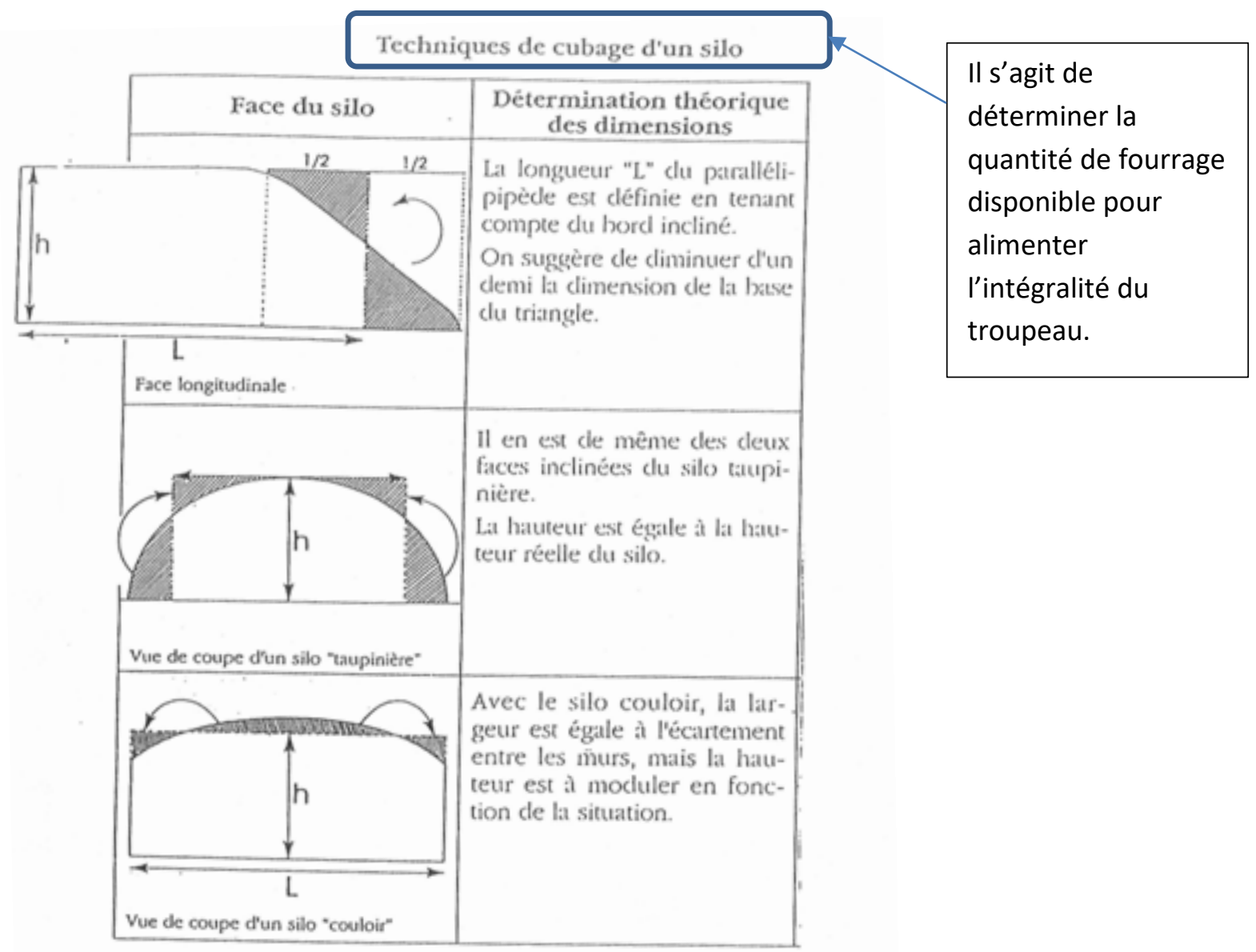

Le support se clôt par un plan de rationnement alimentaire prévisionnel vierge à compléter où les stocks calculés sont comparés aux stocks consommés sous la forme d'un tableau de bilan fourrager (cf. annexe). Là encore, l'exercice relève davantage du travail de gestion d'un exploitant qui se demande s'il aura assez de fourrage pour passer l'hiver, et en ce sens le support ne vise qu'indirectement les objectifs du référentiel, d'où le risque d'une opacité pour les élèves. Ce dernier sous-thème clôt la construction logique de 
l'ensemble des documents présentés. Il constitue en réalité le principal objectif. Il s'agit d'évaluer des stocks d'aliments au regard de ce que les animaux consomment et de séparer l'alimentation du cheptel laitier de l'ensemble du cheptel, ainsi que de déduire quelle est la spécificité de l'alimentation du cheptel laitier au travers des modes de rationnement, par exemple. Les supports se lisent à partir de l'objectif général, fixé tardivement en dernière page (et non progressivement sous-thème par sous-thème), en lien avec des préoccupations pragmatiques que l'on peut formuler ainsi : l'exploitation dispose-t-elle de fourrages en quantité suffisante pour tenir une année ? Les besoins et les stocks sont-ils à l'équilibre, excédentaires, déficitaires? Qu'en est-il de l'alimentation des vaches laitières comparativement aux vaches non laitières ? Cette construction cognitive attendue n'est jamais pleinement consignée dans l'écrit et repose sur l'interprétation du tableau final. La transposition de la pratique professionnelle au contexte scolaire est ici présupposée sans qu'elle ne constitue un objet pédagogique explicite. On peut toujours supposer qu'elle soit un objet d'apprentissage dans le cadre d'un autre enseignement, bien qu'on n'en ait pas vu la trace.

Les supports de l'école 2 : initier aux démarches de raisonnement. Les supports de l'école 2 sont caractérisés par le découpage d'une démarche de raisonnement, laquelle prend la forme d'une séquence continue entre différents types de ressources cognitives à mobiliser par les élèves : recherche d'informations sur l'exploitation-comparaisonsinterprétations-résultats. Le support est organisé en deux grandes parties : la première relève du contexte général de l'exploitation ; la seconde, plus longue, est centrée sur la notion de l'alimentation du troupeau laitier. Autrement dit, le thème principal d'apprentissage est rendu plus visible. Dans cette seconde partie, les élèves sont incités à entrer dans une activité de comparaison des valeurs alimentaires des fourrages (le calcul de ces valeurs par exploitation est souvent réalisé par un service de la Chambre d'Agriculture) et de les mettre en regard avec les pratiques culturales des exploitants. Il s'agit de produire à partir d'un exercice, où n'entre pas toute la complexité du réel, un texte argumenté en s'appuyant sur des documents de la Chambre d'Agriculture (des valeurs alimentaires faibles pouvant s'expliquer par les contraintes d'environnement). 


\section{Extrait 3.}

Ecole 2 : l'organisation de l'exploitation et le rationnement du troupeau laitier

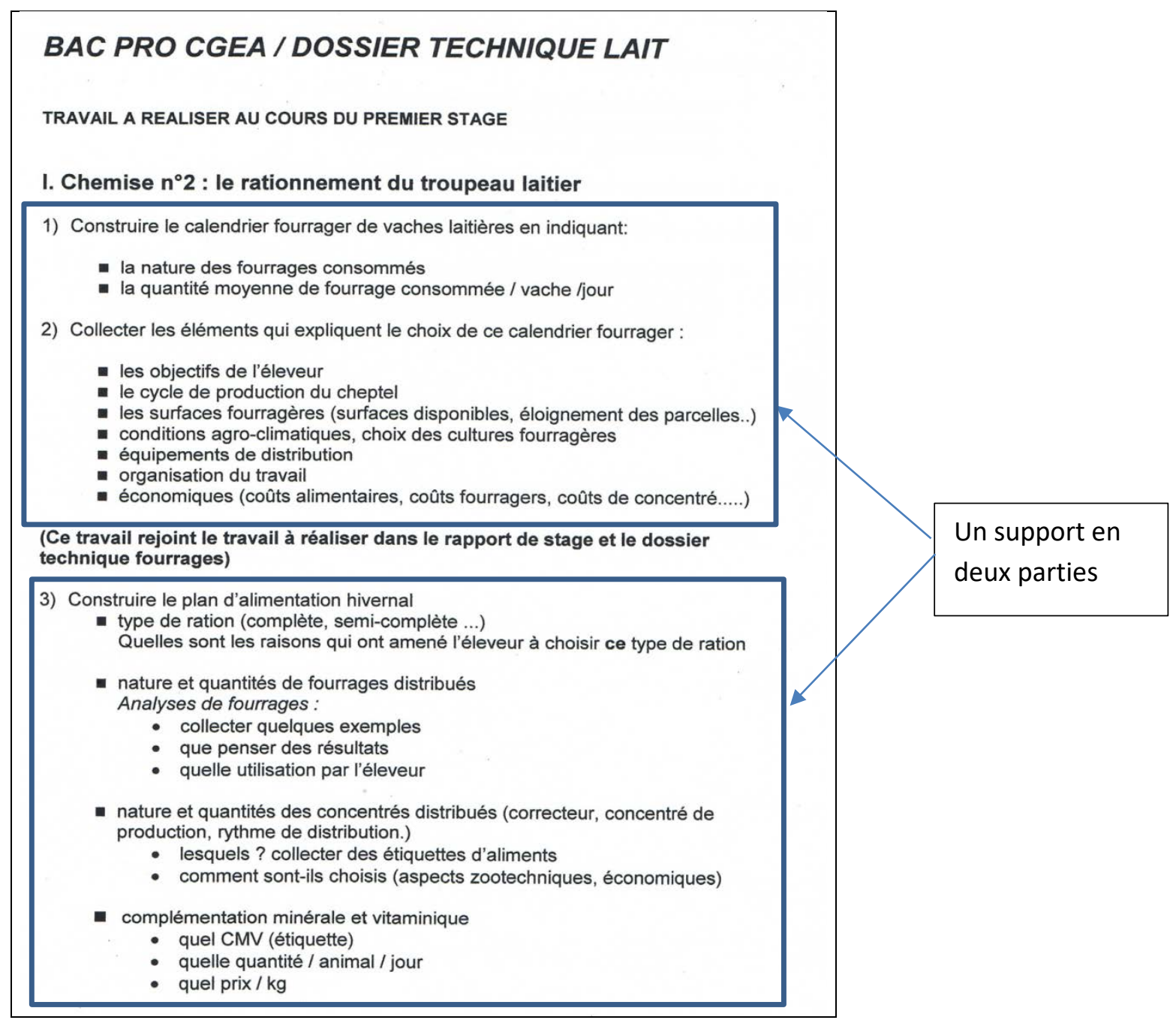

Les pratiques d'éleveurs font aussi objet d'analyse. Différentes interprétations possibles sont alors attendues sur la conduite de l'exploitation. L'une consiste à mettre en rapport les types de culture avec la ration distribuée et ses effets potentiels sur la production 
laitière en procédant à l'évaluation entre ration et la couverture des besoins alimentaires des animaux. Cette interprétation se double de la prise en compte des effets de la ration sur la santé et le métabolisme du troupeau. Enfin, il est demandé de comparer les couts que représente le poste de l'alimentation sur l'exploitation du stage en s'aidant de moyennes d'exploitation pour objectiver des résultats et des pratiques, et se faisant d'indiquer d'éventuelles singularités d'exploitation, de réaliser un état des lieux sur les plans techniques et économiques. Ces différentes attentes (recherche d'information-hypothèsesrésultats-interprétations) forment un schéma qui relève d'une démarche de raisonnement, voire d'une démarche scientifique transposable et adaptable à d'autres situations. En ce sens, l'école 2, plus proche des attendus du référentiel, fabrique chez les élèves une structuration de la pensée en correspondance avec la figure de l'ingénieur agronome. La science agronomique est appliquée à un cas, par opposition au support centré sur l'exploitation dans l'école 1 où, parce qu'il faut répondre à des problèmes dans l'ici et maintenant, la science est reléguée en arrière-plan. Cette opposition nette a d'ailleurs été objectivée par Trabal au sujet de l'enseignement des mathématiques (Trabal, 1996).

\section{Extrait 4.}

Ecole 2 : apprendre à utiliser de l'information

\begin{tabular}{|c|c|c|}
\hline $\begin{array}{l}\text { I. Chemise } n^{\circ} \mathbf{2} \text { : le rationnement du troupeau laitier } \\
\text { 1) Construire le calendrier fourrager de vaches laitières en indiquant: } \\
\text { - la nature des fourrages consommés } \\
\text { - la quantité moyenne de fourrage consommée / vache /jour }\end{array}$ & 4 & \begin{tabular}{|l} 
Apprendre à \\
collecter de \\
l'information
\end{tabular} \\
\hline $\begin{array}{l}\text { 2) Collecter les éléments qui expliquentle choix de ce calendrier fourrager: } \\
\text { - les objectifs de l'éleveur } \\
\text { - le cycle de production du cheptel } \\
\text { - les surfaces fourragères (surfaces disponibles, éloignement des parcelles..) } \\
\text { - équitions agro-climatiques, choix des cultures fourragères } \\
\text { - organisation du distribution } \\
\text { - économiques (coûts alimentaires, coûts fourragers, coûts de concentré.....) }\end{array}$ & 4 & $\begin{array}{l}\text { Apprendre } \\
\text { à utiliser } \\
\text { l'informatio } \\
\text { n collectée } \\
\text { pour } \\
\text { construire } \\
\text { un texte. }\end{array}$ \\
\hline
\end{tabular}

Les supports produits dans l'école 2 témoignent d'une hybridation spécifique du travail agricole et de l'école. Le métier est transformé en un objet de scolarisation, il devient ce par quoi les élèves doivent se conformer à une méthode d'appropriation des savoirs et d'argumentation. En d'autres termes, le métier devient enseignable, énonçable, partageable (Dubar \& Tripier, 1998). Les différences entre les supports s'étendent à l'activité scolaire de rédaction. En effet, alors que l'activité scripturale attendue des élèves était réduite au profit d'indicateurs dans l'école 1, elle devient l'activité principalement visée par les documents de l'école 2. Les supports de l'école 2 contiennent des consignes écrites longues, quand dans l'école 1 le style phrastique était averbal et plutôt télégraphique. La formulation explicite des contenus attendus oblige à dépasser l'écriture simple d'indicateurs pour construire un texte avec ces derniers (dans une autre partie du support pédagogique de l'école 2, on trouve les questions suivantes : "Quelles sont les raisons qui 
ont amené l'éleveur à choisir ce type de ration ?", "que penser des résultats ?", " comment (les concentrés) sont-ils choisis ? ", cf. annexes).

Il s'agit donc de produire un texte en son entier, c'est-à-dire de construire un écrit cohérent, long, en lien avec une méthode de raisonnement (décrire des résultats, en donner une interprétation au regard de contraintes environnementales, évaluer les valeurs alimentaires sur la production laitière, étudier les incidences techniques et économiques de la ration). Qu'en est-il alors des productions écrites des élèves ?

\section{Les dossiers des élèves}

Si les deux approches pédagogiques sont intéressantes, parfois de très haut niveau cognitif, elles s’adressent à des élèves qui y sont inégalement préparés, d'où certaines ambiguïtés et difficultés d'apprentissage retrouvées dans les écrits des élèves. Les spécificités des savoirs et des logiques pédagogiques (centrée directement sur l'activité productive dans l'école 1 ; davantage scolarisée dans l'école 2) produisent chez des interprétations différenciées parfois éloignées de celles attendues.

Des littératies locales dans l'école 1. Si dans l'école 1, les élèves font preuve d'une volonté évidente de réussir scolairement alors que leur parcours antérieur est ponctué par l'échec, ils peinent cependant à interpréter ce qui convient de faire et comment. Dans l'écrit ci-dessous, sur le plan de l'organisation des arguments, l'élève expose les objectifs supposés d'une pratique d'élevage, cherche à en construire des indices de sens (les textes des élèves sont reproduits fautes comprises) :

\section{Extrait 5.}

Ecrits des élèves. Les objectifs de l'éleveur

« Les objectifs des l'éleveurs

Tout d'abord, le principal objectif est de produire le quota ainsi que les augmentations que la laiterie donne.

Pour ce qui est de la productivité de l'élevage, l'exploitant recherche :

- au niveau génétique : mamelle, morphologie, fertilité, lait et taux

- au niveau production : recherche un niveau de production à 8500-9500/VL/an

- favoriser le pâturage

-maintenir le maïs toute l'année

- garder suffisamment de blé pour l'autoconsommation » (Bastien).

Ce type d'écrit situé au début des deux années de formation observées témoigne d'un effort de structuration avec la formulation d'une phrase introductive, et qui hiérarchise « un principal objectif » et le reste «pour ce qui est... ». La suite est constituée d'une liste plus hétérogène dont les niveaux ne sont pas ordonnés. La consigne d’introduire est donc suivie, mais l'attente de hiérarchisation des objectifs est ensuite perdue de vue. L'auteur de ces lignes, Bastien, est plutôt un bon élève du groupe-classe. De père ouvrier et mère employée, il est issu de seconde générale et avait pour projet initial de devenir vétérinaire. Ses difficultés rencontrées en seconde ont cependant fait obstacle à une orientation en première scientifique, raison pour laquelle il bifurque d'abord en BEP Agricole, puis en 
bac pro CGEA, avec l'espoir cette fois de devenir exploitant agricole. Il est un des rares élèves du groupe-classe à poursuivre sa scolarité en BTS Agricole.

Plus tard dans la formation, il ne semble pas que cette difficulté à organiser l'écrit, à élaborer et conserver un fil conducteur soit réduite pour l'ensemble des élèves. Au contraire, les difficultés s'accentuent pour une bonne part de la classe. Ainsi, le mode descriptif faiblement hiérarchisé montre combien ils deviennent prisonniers d'un contexte local et restreint (extraits 6 et 7).

\section{Extrait 6.}

Ecrits des élèves. Aller voir ses vaches vers $22 \mathrm{H}$

« Tout les soirs l'agriculteurs vient voir ses vaches (vers 22h) pour repérer les vaches en chaleur. » (Erwin).

« Repérage des chaleurs : ils sont tous repérés grâce à la vue, et si les vaches sont en chaleur, on regarde sur le bulletin du contrôleur laitier si elles sont bonnes à inséminer » (Laurent).

« (...) l'éleveur à sa maison à côté, se qui permet de surveiller aisément, les mises bas » (Jenny).

« Les vêlages ont le plus souvent lieu au pré, avec une observation régulière. Les vêlages se font sans aide, lorsque tout se présente bien. L’éleveur préfère laisser faire la nature » (Jenny).

« La surveillance des chaleurs est également réalisée le matin, le midi et le soir, en restant avec les animaux environ 15 minutes » (Valentyn).

\section{Extrait 7.}

Ecrits des élèves. Gestion de la reproduction

Les éleveurs ne font pas de synchronisations mai font le regroupage des chaleurs ${ }^{2}$ (Pascal).

\footnotetext{
${ }^{2}$ Pascal énonce l'existence d'un « regroupage », sans comprendre que cette action signifie que le troupeau est synchronisé notamment par le recours à un traitement hormonal. Les mêmes confusions sont à l'œuvre pour Jérôme, si les vêlages sont groupés, c’est parce qu’il existe au préalable une synchronisation des animaux en vue d'une rationalisation du travail au moment des chaleurs et donc des inséminations comme au moment des vêlages. Il en va de même pour Jenny. Il énonce que la synchronisation se réalise chez les primipares (c’est-à-dire les vaches qui n’ont jamais vêlées) au moment de leur entrée dans le troupeau. En toute logique le troupeau fait donc l'objet d'une synchronisation.
} 
Les vêlages sont groupés, mais aucune synchronisation n'est utilisée (Jérôme).

il n’y a pas de synchronisation sur les multipares mais sur les primipares (Jenny).

Sur le plan du contenu et de la référence à l'expérience de métier, les observations issues de la période de stage et les propos du maître de stage sont rapportés de manière brute et littérale et non insérées dans une logique de mise à distance des observations empiriques ou de hiérarchisation des objectifs. C'est donc sur le mode du récit que sont rapportées la topographie des lieux et la soumission aux contraintes temporelles cycliques, deux traits culturels traditionnels du rapport à la tâche de l'agriculteur desquels le référentiel vise à rompre. Les lieux sont manifestés par "la maison à côté » et "au pré ». Le temps cyclique est rythmé par l'heure, le matin, le midi, le soir et par le cycle de gestation de la vache. Les acteurs sont moins définis par leur fonction propre ou par leur coopération dans un collectif de travail que par leurs attaches aux lieux (l'éleveur et sa maison, l'éleveur " laisse faire la nature » quand le vêlage a lieu « au pré ») ou à l'heure/la durée (50 jours et le contrôle de l'inséminateur et du vétérinaire, le soir « en restant avec les animaux environ 15 minutes »). Les textes des élèves sont une suite de phrases générales, dont les liens ne sont pas toujours évidents à percevoir. Tout se passe comme si ces derniers avaient compris la nécessité de mettre ensemble des objets, mais que ne sachant lesquels, ils mobilisent des éléments sans établir de rapports entre eux, passant de procédures à des trucs et astuces pratiques : "Le repérage des chaleurs se fait en repoussant les refus ${ }^{3}$ ". L'absence de mise en relation avec des savoirs entraîne pour ces élèves une impossibilité de mettre en œuvre un raisonnement porteur d'une généricité, empêche la mobilisation de ressources pour penser sur, pour parler sur, fonde une des causes explicatives pour laquelle les élèves disent la pratique sans s'en distancier.

Toutefois, deux élèves sur quinze fournissent des écrits plus distanciés quant au contenu et plus organisés quant à la forme. Ces deux élèves, Bastien et Jérémie ont ceci de particulier qu'ils proviennent pour l'un du lycée général et pour l'autre de l'école 2 étudiée. En effet, un des éléments qui distingue l'écrit de Jérémie des autres élèves de la classe relève de sa familiarité avec la mise en œuvre de procédés de rédaction typiques de l'école 2, en particulier la mise en évidence d’écarts. Pourtant considéré comme « mauvais » par les enseignants de l'école 2, il a été incité à quitter cet établissement, et s'est inscrit dans l'école 1. Pourtant, du point de vue de l'écrit, il maîtrise, dans ses manières de faire des formes de raisonnement, qui justement l'émancipe d'un contexte par trop étroit grâce à une méthode de pensée reproductible et transposable que n'enseigne pas l'enseignant de l'école 1. On voit donc nettement se dessiner l'empreinte des pédagogies distribuées et de leurs effets potentiels sur les élèves.

Les écrits des élèves de l'école 2. Quant au contenu, les écrits des élèves de l'école 2 fournissent les traces d'une mise à distance des situations par les problèmes qu'elles posent. La forme de l'écriture s’inspire plutôt du modèle de la science appliquée.

Extrait 8.

3 « les refus », c’est-à-dire les aliments que les vaches ont refusé d’ingérer.

\begin{tabular}{llc}
\hline \hline Language and Literacy & Volume 20, Issue 1, 2018 & Page 138
\end{tabular}


Ecrits des élèves. Montrer des écarts

«Tout d'abord l'intervalle vêlage 1ère IA (première Insémination Artificielle) paraît réparti à près de 50 \% sur les données supérieur à 90 jours (après le vêlage) on pourrait se poser la question y a-t-il un problème de mise à la reproduction (mauvaise détection de chaleurs, déficit énergétique ou problème sanitaire). En fin de compte le pourquoi du comment reste facile à expliquer l'éleveur veut mettre ses animaux à la reproduction lorsqu'ils se sont « refaîtes » pour avoir plus de chance de réussite.

L'explication donner au-dessus se confirme car le taux de réussite en $1^{\text {ère }}$ en IA ma paraît très convenable car on a plus de $56 \%$ et plus de $25 \%$ en $2^{\text {ème }}$ IA donc une bonne fertilité du troupeau laitier (Jordenn).

Au-delà des maladresses de formulation, l'écrit atteste d'un pluri-référencement prenant sa source dans les indicateurs retenus par l'élève (le nombre d'inséminations artificielles et la période pendant laquelle elles sont pratiquées), les pourcentages pour rendre compte d'une cohérence entre des éléments inhabituels (la mise en évidence d'un écart), et enfin les propos rapportés du maître de stage. Les différentes options possibles sont restituées en forme de dialogue puis suivie d'une tentative de résolution du problème. Le discours pédagogique de la "situation-problème » revendiqué par l'enseignant semble ici trouver une trace tangible, même si le mode d'expression n'est pas totalement conforme aux normes académiques de l'écriture.

Une littératie de la science appliquée comme amendement au référentiel de formation

Les différences d'appropriation entre les élèves partagent un mode de raisonnement commun à l'ensemble de la classe. Ci-dessous, Pierre et Mathilde tentent de construire des causes possibles aux écarts constatés.

Extrait 9.

Ecrits des élèves. Chercher des causes

« Le pourcentage d'infertilité a augmenté de $10 \%$ en 2008-2009, du à une chute de $32 \%$ des vaches fécondes en 1ère IA. L'intervalle vêlage-vêlage a augmenté de 11 jours en 2008-2009. L’année 2008-2009 est plutôt négative, au niveau des résultats ceux-ci s'explique par les problèmes sanitaires rencontrés sur l'exploitation (...). Pour la réforme, il y a eu une augmentation de $9 \%$ du taux de réforme en 2008 du à des problèmes sanitaires (FCO et paratuberculose) qui ont touchés le troupeau laitier » (Mathilde).

«La part la plus importante des réformes est l'infertilité. Cela peut ce comprendre a un niveau d'étable de $9500 \mathrm{~kg}$ de lait. De plus, les boiteries on a une part importante dans le taux de réforme car il y a une présence de dermatite sur l'exploitation » (Pierre).

Les enchaînements "dû à », " s'explique par », " cela peut se comprendre » et " de plus... car » signalent les imputations de causalité, elle-même explicable par des savoirs mobilisés (infertilité, boiterie, dermatite). Les objets techniques du travail agricole sont mis 
en regard des supports pédagogiques. Cette mise en circulation d'un ensemble de pratiques dont la finalité reste le meilleur rendement possible sous réserve des contraintes constitue un schéma énonciatif qui s’apparente au mode de raisonnement de l'ingénieur : décrire des constats, formuler des hypothèses (mauvaise détection de chaleurs, déficit énergétique ou problème sanitaire), mettre à l'épreuve ses hypothèses, même si ces dernières ne sont pas toutes traitées.

La phase inductive illustrée par la visite et sa préparation et les retours d'expérience du stage au travers des propos du maître de stage éloignent en effet la démarche du modèle des sciences expérimentales descendantes comme la physique4. Les écrits puisent dans l'expérience d'un exploitant agricole pour ensuite en déduire une posture de l'élève s'identifiant à ce responsable d'exploitation. De ce fait, les écrits ne sont pas très académiques, et cela rejoint notre propos de ce texte : ils ne sont pas académiques du fait de la spécificité de la formation professionnelle. Toutefois, ils s'en rapprochent par un mode de construction de la connaissance de surplomb alternant induction, hypothèse et déduction.

\section{Synthèse-discussion-conclusion : l'étude des supports et sa portée heuristique dans l'enseignement professionnel}

\section{Synthèse des résultats}

Les deux pédagogies objectivées permettent d’interroger les effets des littératies sur les apprentissages des élèves. Dans le cas précis, lorsqu'elles sont presque directement transposées du travail productif au contexte scolaire elles sont adressées aux élèves plus souvent en échec à l'école. À l'inverse, elles sont plus classiquement scolaires lorsque les élèves ont été en réussite au collège. Les effets produits sont socialement caricaturaux : les élèves de l'école 1 confrontés à des savoirs pratiques adoptent un mode de pensée localement situé, lorsque ceux de l'école 2 confrontés à des savoirs issus de la science appliquée acquièrent beaucoup plus massivement une attitude de surplomb sur les pratiques quotidiennes.

\section{Discussion}

Certes, l'échantillon de l'étude comporte un nombre trop peu important d'élèves et de classes pour permettre une quelconque généralisation. Toutefois ces études de cas autorisent à construire l'hypothèse que la question des littératies et des inégalités ne cesse pas au seuil de l'enseignement professionnel, mais au contraire s'y perpétue autrement.

D’ailleurs, les types de pédagogies retrouvées convergent vers d'autres travaux. Eric Plaisance (1986) avait mis en lumière des mêmes procédés dès l'école maternelle. Même si l'auteur n'utilise pas le concept de littératie, ses recherches montrent l'existence d'une distribution de savoirs. Les "savoirs expressifs" marqués par des activités d'explicitation, d'argumentation seraient plus souvent présentés aux élèves héritiers de la culture scolaire et les savoirs "productifs » (id.), marqués par le sens utile et manuel des savoirs, dédiés à ceux dont les pratiques culturelles en sont plus éloignées. Les mêmes logiques ont été mises en évidence par le travail historique de Harlé (2009) : les classes d'Enseignement Manuel Technique (EMT) «petites usines dans l'école » (Haby in Harlé,

\footnotetext{
${ }^{4}$ On trouve ici une trace des débats entre partisans d'un enseignement pratique-industriel de la technique au collège et partisans, physiciens pour la plupart, d’un enseignement « technologique » (Harlé, 2009). 
2009) avaient été conçues à la fin des années 1970 pour les élèves en difficultés scolaires (souvent d'origine populaire) au moment de la mise en place du collège unique (ce qui constituait d'ailleurs un indicateur d'une non-unification du collège). Enfin, les travaux de l'équipe EScol, parmi beaucoup d'autres, mettent en évidence la perpétuation de tels phénomènes dans tous les cycles de l'enseignement général (Bautier et al., 2012 ; DelarueBreton, 2012 ; Kakpo, 2012 ; Rochex et Crinon, 2011).

\section{Conclusion}

Dans cette perspective, les principales conclusions de notre analyse suggèrent que malgré des spécificités propres, les logiques scolaires ici retrouvées dépassent l'enseignement agricole. Pour conforter ou réfuter cette hypothèse il faudrait alors privilégier l'analyse d'autres classes de l'enseignement professionnel et favoriser les modes relationnels d'étude alliant différents segments du système éducatif pour construire une connaissance plus élargie du système éducatif.

\section{Bibliographie}

Balcou-Debussche M. (2016). De l'éducation thérapeutique du patient à la littératie en santé, Problématisation socio-anthropologique d'objets didactiques contextualisés. Paris : Vrin.

Barton D., \& Hamilton M. (2010). La littératie : une pratique sociale. Langage et société, 3(133), 45-62.

Bautier É., Crinon J., Delarue-Breton C. \& Marin B (2012). « Les textes composites : des exigences de travail peu enseignées ? », Repères, 45, 63-79.

Bautier É., \& Rochex J-Y. (1998). L'expérience scolaire des «nouveaux lycéens ». Démocratisation ou massification ?. Paris : Armand Colin.

Bautier É. (1995). Pratiques langagières, pratiques sociales. Paris : L’Harmattan.

Beaud, S. (2003). 80 \% au bac... et après : Les enfants de la démocaratisation scolaire. Paris: La Découverte.

Benoît H., Ebel M., \& Fiala P. (1984). Sous le consensus, la xénophobie. Mots, 8(1), 213216.

Bonnéry, S. \& Joigneaux, C. (2015). Des littératies familiales inégalement rentables scolairement. Le français aujourd'hui, 190,(3), 23-34.Boutet, J. (2001). La part langagière du travail : bilan et évolution. Langage et société, 98(4), 17-42.

Boutet J. (1980). Quelques courants dans l'approche sociale du langage. Langage et société, 12(2), 33-70.

Boutet J., Gardin B., \& Lacoste M. (1995). Discours en situation de travail. Langages, 117, 68-85.

Butault J.P., \& Delame N. (2003). La disparition des exploitations s'accélère sans concentration excessive. Agreste, 3, 17-26.

Denis B., \& Theret M. (1994). Les grands traités de zootechnie et leur conception de cette discipline. Ethnozootechnie, 54, 3-24.

Delarue-Breton C., \& Bautier, É. (2015). Nouvelle littératie scolaire et inégalités des élèves : une production de significations différenciée. Le français aujourd'hui, 190(3), 51-60. 
Delarue-Breton, C. (2012). Discours scolaire et paradoxe. Louvain-la-Neuve, Belgique, Éditions Academia Bruylant.

Dubar C. \& Tripier P. (1998). Sociologie des professions, Paris, A. Colin, 1998, coll. «U $»$.

Glaser, B.G., \& Strauss, A.L. (1967). The Discovery of Grounded Theory: Strategies for Qualitative Research. Chicago : Aldine.

Fraenkel, B. \& Mbodj-Pouye, A. (2010). Introduction: Les New Literacy studies, jalons historiques et perspectives actuelles. Langage et société, 133,(3), 7-24.

Harlé I. (2009). La fabrication des savoirs scolaires. Paris : La dispute.

Heath S.-B., \& Street B. (2008). On Ethnography: Approaches to Language and Literacy Research, Language \& Literacy (NCRLL). New-York : Teachers College Press.

Hennion A. (2013). "D'une sociologie de la médiation à une pragmatique des attachements », SociologieS [En ligne], Théories et recherches, mis en ligne le 25 juin 2013.Hennion A. (2007 [1993]). La Passion musicale. Paris : Métailié.

Kakpo, S. (2012). Les devoirs à la maison : Mobilisation et désorientation des familles populaires. Paris: Presses Universitaires de France.Laparra, M., \& Margolinas, C. (2012). Oralité, littératie et production des inégalités scolaires. Le français aujourd'hui, 177(2), 55-64.

Lahire B. (1995). Tableaux de familles : heurts et malheurs en milieux populaires. Paris : Seuil.

Latour B. \& Woolgar S. (1988). La Production des faits scientifiques. Paris: La Découverte, «Sciences et société ».

Masny, D., \& Dufresne, T. (2007). Apprendre à lire au 21e siècle, in : A.M. Dionne \& M.J. Berger (Eds.), Les Littératies : perspectives familiale, linguistique et culturelle (pp. 209-224). Ottawa: Les Presses de l'Université d'Ottawa.

Papen, U. (2010). Literacy mediators, scribes or brokers: The central role of others in accomplishing reading and writing. Langage et société, 133,(3), 63-82.

Plaisance É. (1986). L’enfant, la maternelle, la société. Paris : Presses Universitaires de France.

Référentiel de formation bac pro CGEA, (2017) http://www.chlorofil.fr/diplomes-etreferentiels/formations-et-diplomes/baccalaureat-professionnel/conduite-etgestion-de-lentreprise-agricole.html

Rochex J-Y \& Crinon J. (dir.). (2011). La construction des inégalités scolaires. Au cœur des pratiques et des dispositifs d'enseignement : Rennes : Presses universitaires de Rennes.

Rockwell, E. (2010). L'appropriation de l'écriture dans deux villages nahua du centre du Mexique. Langage et société, 133,(3), 83-99.

Scribner, S., \& Cole, M. (1973). Cognitive Consequences of Formal and Informal Education. Science, 82, 553-559.

Trabal, P. (1996). Le sens commun, les mathématiques et les sciences. Une approche de la sociologie des sciences par une étude des représentations sociales des mathématiques et des sciences. Paris : École des hautes études en sciences sociales (EHSS).

Viriot-Goeldel, C., \& Delarue-Breton, C. (2014). Des textes composites à l'école : nouvelle littéracie scolaire, apprentissages et inégalités. Spirale. Revue de recherches en éducation, 53, 21-31. 


\section{Biographie de l'auteure}

Laure Minassian. Docteur en sociologie de l'éducation, chercheure au laboratoire CIRCEFT-EScol (Centre Interdisciplinaire de Recherche Culture Éducation Formation Travail - Education et scolarisation) de Paris VIII, sa thèse porte sur les inégalités sociales et scolaires dans l'enseignement agricole. Elle exerce actuellement la fonction d'Attachée Temporaire à l'Enseignement et à la Recherche à l'ESPE (Ecole Supérieure du Professorat et de l'Education) à La Réunion. 


\section{Annexes}

\section{BAC PRO}

\section{REALISATION D'UN BILAN FOURRAGER}

Au printemps, à partir des surfaces mises en place (= assolement fourrager) il est prévu de récolter des fourrages pour l'hiver : foin, ensilages d'herbe ou maïs, paille, choux ...

A l'automne, ces fourrages sont stockés, et les stocks qui correspondent plus ou moins aux prévisions doivent être contrôlés et confrontés aux besoins du cheptel, en établissant des rations. On peut alors comparer les stocks disponibles et les besoins du cheptel. Cette démarche, appelée BILAN FOURRAGER, comprend 4 étapes :

\section{1) L'inventaire des stocks fourragers.}

Il se réalise soit par :

- CUBAGE pour les silos (ensilages, betteraves ...) avec l'appréciation de la densité pour calculer le tonnage.

- COMPTAGE pour les stocks de foin, paille, en bottes ou balles.

- PESEE d'échantillons (pesée géométrique) pour les fourrages encore sur pied (choux ...)

\section{2) L'inventaire des LOTS D'ANIMAUX}

Groupés par catégories, en fonction de :

objeciiŕs de production

. stade physiologique

. âge, poids ...

\section{3) Un Plan de RATIONNEMENT pour chacun des lots (voir fiche)}

Ce plan de rationnement est à envisager sur 12 mois, mais la date de fin sera variable pour chaque fourrage, selon la date de la prochaine récolte.

Ex : . 15/04 pour ensilage d'herbe

. 15/06 pour foin

$.15 / 10$ pour ensilage maïs.

\section{4) LE BLLAN proprement dit}

= Comptabiliser les besoins pour chaque fourrage (en tenant compte de tous les lots) pour comparer aux stocks disponibles. 


\section{BAC PKO}

\section{REAIISATION D'UN BILAN FOZRRAGER}

Au priniemps. à pautir des suffaces mises eu place (= assolement fuurrager) il est prévu de rócolter des fnurrages pour l'hiver : fnin, ensilgges d'herbe ou maïs, paille, choux ...

A l'automac, ces fortrages sont stockés, et les stocks qui coriespoadent plus ou moins aux prévisious doivent être contrúlés et conftontés aux besnins du cheptel, en étzblissant des rations. On peut alors comparer les stocks disponibles et les besoias du cheptel. Citte Atmacic, appelec BII.AV FOURRAGER, comprcud 4 stapes :

\section{LLimentaire des stocks fourrakers}

Il se réalise soit par :

- CUBACE pour les silos (ensilages, betteraves ...) avec l'sppróciation de la densité pour calculer le tonnaye.

- COMPTACIE pour les stocks de foin, pailte, en bottes on balles.

- PESEE d'échantillons (pesée géımétrig̨e) puuz les fuu:rạ̧es enco:e sur pied (choux ...)

\section{2ЦLimentaire des XORS D'AATMAOX}

Groupés par caléguries, en fonction de :

. objectifs de procuction

- stade physiologique

. sge, paids ...

\section{3i. Vin Plan de RATHONNEWENT pour chacun des lots (vorir fiche)}

Ce plaa de ratiounement est à envisage: su: 12 mois, tmais la date de fin sera variable pour chaque fisurrage, selan ia date de Is prochaiue técolte.

Hx: . 15:04 porc eusilage otherbe

. 15,166 pour foin

15:10 pour ensilage mais.

\section{S.LE BILAN proprement dit}

= Comptabiliser les becoins pour cbaçue fourage (ea tevant compte óc tous les lots) pour comparer aux stucks cisponibles. 


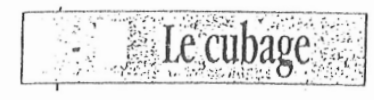

Techniques de cubage d'un silo
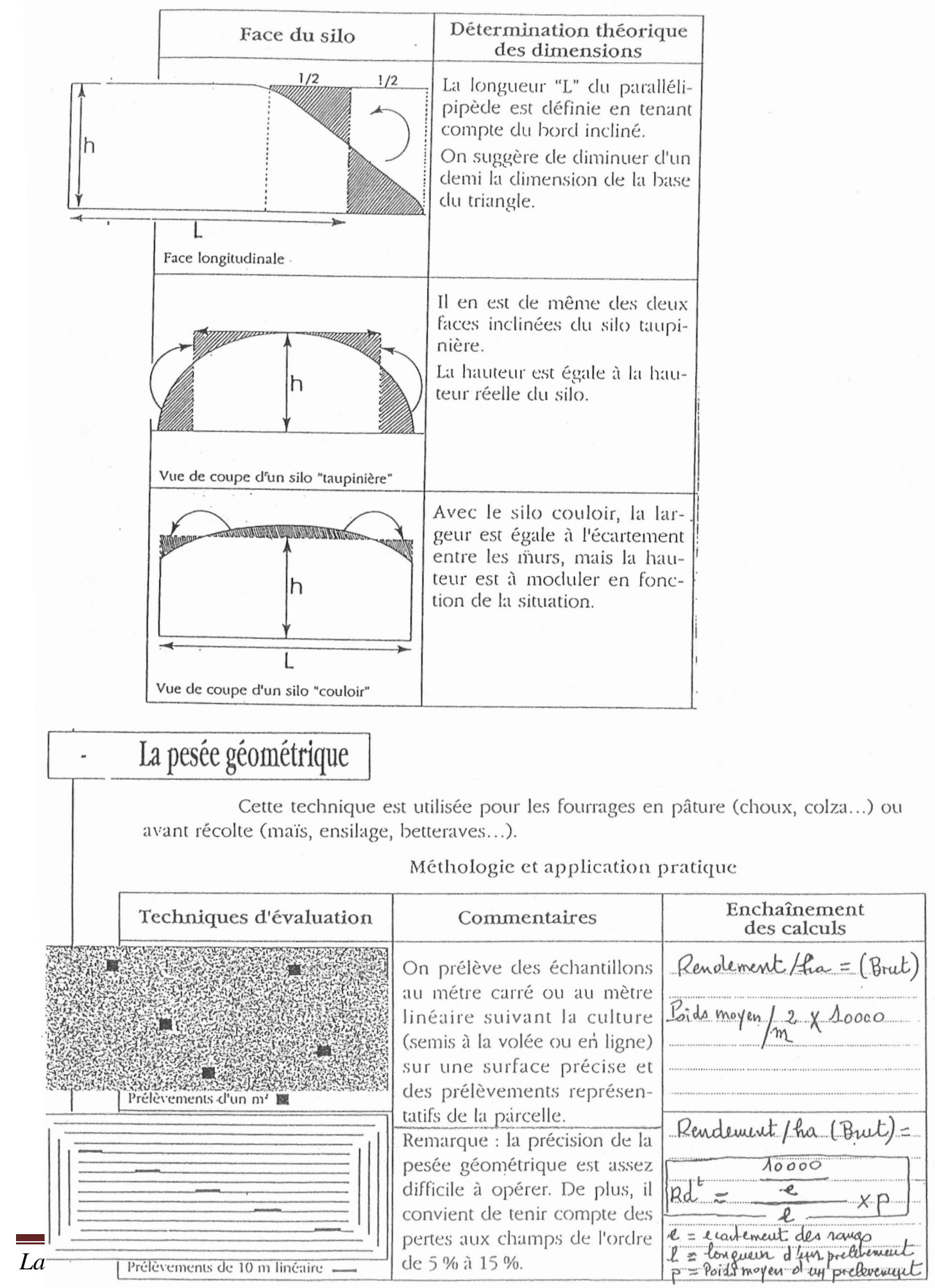

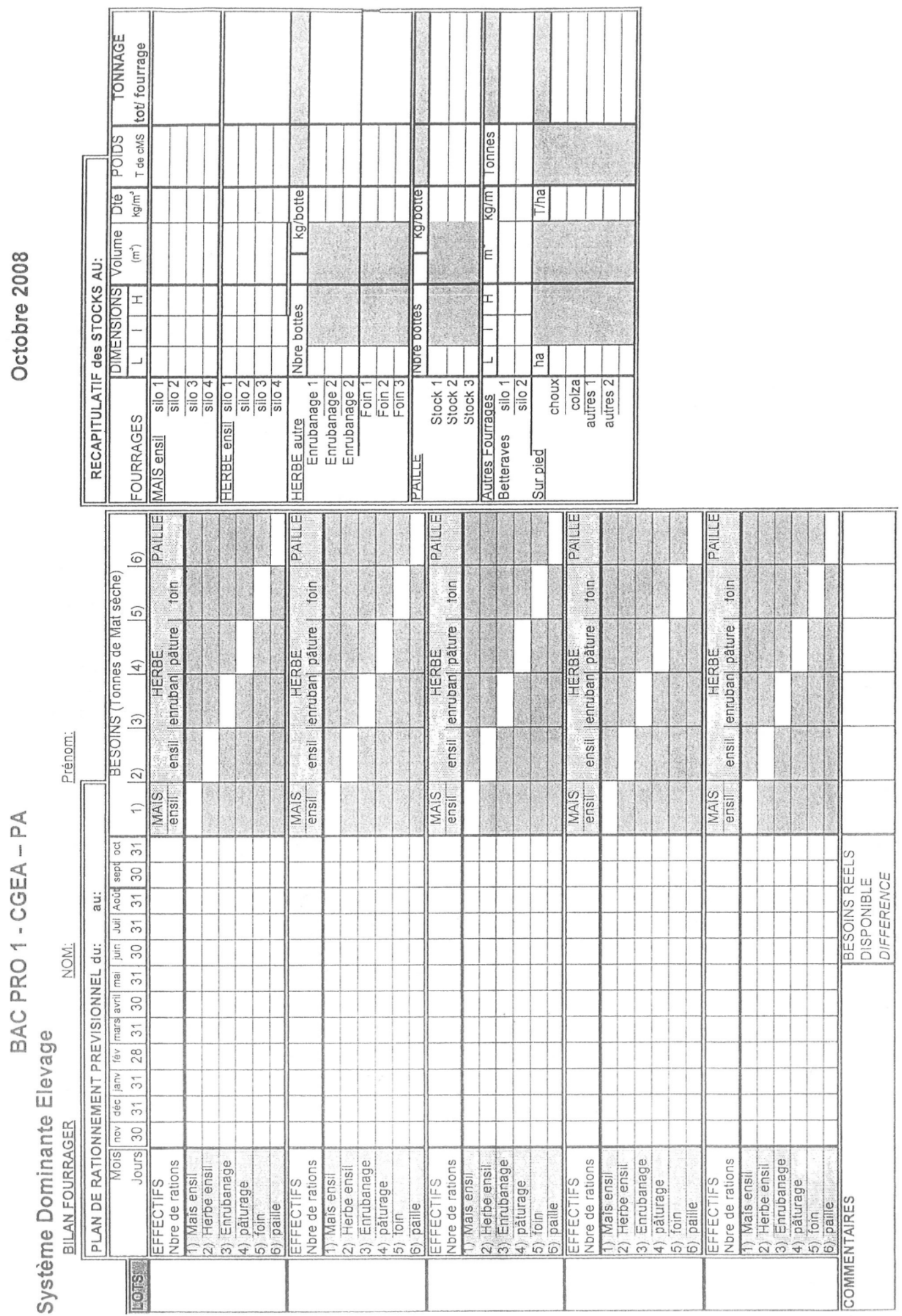